EPJ Web of Conferences 20, 03005 (2012)

DOI: $10.1051 /$ epjconf/20122003005

(C) Owned by the authors, published by EDP Sciences, 2012

\title{
Asymmetric dense matter in holographic QCD
}

\author{
Youngman $\mathrm{Kim}^{1,2, \mathrm{a}}$, Yunseok Seo ${ }^{3, \mathrm{~b}}$, Ik Jae Shin ${ }^{1, \mathrm{c}}$, and Sang-Jin $\operatorname{Sin}^{4, \mathrm{~d}}$ \\ 1 Asia Pacific Center for Theoretical Physics, Pohang, Gyeongbuk 790-784, Republic of Korea \\ 2 Department of Physics, Pohang University of Science and Technology, Pohang, Gyeongbuk 790- \\ 784, Korea \\ 3 Center for Quantum Spacetime, Sogang University, Seoul 121-742, Korea \\ ${ }^{4}$ Department of Physics, Hanyang University, Seoul 133-791, Korea
}

\begin{abstract}
We study asymmetric dense matter in holographic QCD. We construct asymmetric dense matter by considering two quark flavor branes with different quark masses in a D4/D6/D6 model. To calculate the symmetry energy in nuclear matter, we consider two quarks with equal masses and observe that the symmetry energy increases with the total charge showing the stiff dependence. This behavior is universal in the sense that the result is independent of parameters in the model. We also study strange (or hyperon) matter with one light and one intermediate mass quarks. In addition to the vacuum properties of asymmetric matter, we calculate meson masses in asymmetric dense matter and discuss our results in the light of in-medium kaon masses.
\end{abstract}

\section{Introduction}

Asymmetric dense matter is one of the most interesting topics in modern nuclear physics. In general, Nature provides isospin asymmetric nuclear matter in the interior of a nucleus with unequal number of protons and neutrons. In view of larger scale, neutron star is the well-known object we can find. This asymmetric circumstance could also be realized in the heavy ion collisions with isotope.

The nuclear symmetry energy is a core quantity on studying the asymmetric nuclear matter. Even though it has been studied long times, still it remains many things to understand. Furthermore, for the supra-saturation region, there is no consensus in the community whether it shows stiff or soft density dependence. For a review and a recent discussion about the symmetry energy, see [1-7].

Another interesting behavior in an asymmetric dense system is the splitting of hadron masses [810]. In dense medium, the masses of mesons with different isospins behave in different ways. One of the resultant example for this is $K^{-}$condensation.

Here we will use the holographic QCD model to study these properties of asymmetric dense matter. Recent developments based on the AdS/CFT correspondence give new tools to look into the strong coupling regime [11-13]. Even though there exist many differences from reality, the effort which applies this correspondence to QCD progresses attaining some successful results [14-16].

\section{Model: D4/D6/D6 system}

In dense matter, as the density increases, the transition occurs from the nuclear to strange matter. In a low density regime, there exist only the light quarks ( $u$ and $d$ ) because the strange quark mass is

\footnotetext{
a e-mail: ykim@apctp.org

b e-mail: yseo@sogang.ac.kr

c e-mail: geniean@apctp.org

d e-mail: sjsin@hanyang.ac.kr
}

This is an Open Access article distributed under the terms of the Creative Commons Attribution-Noncommercial License 3.0, which permits unrestricted use, distribution, and reproduction in any noncommercial medium, provided the original work is properly cited. 


\section{EPJ Web of Conferences}

much larger than that of light ones. As the density increases, however, the chemical potential of light quarks also increases and becomes comparable to the mass of strange quark. Then it starts to pile up the intermediate mass quarks (s). The existence of quarks with different masses can give asymmetry in dense matter.

The model we will use here is a D4/D6/D6 system with compact D4 branes as baryon vertices [17]. In usual D4/D6 system, according to the AdS/CFT dictionary, the distance between D4 and probe D6 branes corresponds to the quark mass. To describe the system with two different masses, we will put two distinguished D6 branes with different asymptotic heights. In this section, to understand the structure of this model, we will briefly review the paper [17].

We start from the expression of the confining D4 background metric.

$$
\begin{aligned}
d s^{2} & =\left(\frac{U}{R}\right)^{3 / 2}\left[-d t^{2}+d \mathbf{x}^{2}+f(U) d x_{4}^{2}\right]+\left(\frac{R}{U}\right)^{3 / 2}\left[\frac{d U^{2}}{f(U)}+U^{2} d \Omega_{4}^{2}\right] \\
& =\left(\frac{U}{R}\right)^{3 / 2}\left[-d t^{2}+d \mathbf{x}^{2}+f(U) d x_{4}^{2}\right]+\left(\frac{R}{U}\right)^{3 / 2}\left(\frac{U}{\xi}\right)^{2}\left(d \xi^{2}+\xi^{2} d \Omega_{4}^{2}\right) \\
e^{\Phi} & =g_{s}\left(\frac{U}{R}\right)^{3 / 4}, \quad F_{4}=\frac{2 \pi N_{c}}{\Omega_{4}} \epsilon_{4} \quad \text { where } f(U)=1-\left(\frac{U_{K K}}{U}\right)^{3}
\end{aligned}
$$

This is related to the black D4 brane by the double Wick rotation. Here the dimensionless coordinate $\xi$ is used.

$$
\left(\frac{U}{U_{K K}}\right)^{3 / 2}=\frac{\xi^{3 / 2}}{2}\left(1+\frac{1}{\xi^{3}}\right)=\frac{\xi^{3 / 2}}{2} \omega
$$

In the holographic QCD model, a baryon is expressed by instanton or compact D branes wrapping on spherical volume through transverse directions [18]. In D4/D6/D6 the compact D4 brane could be interpreted as baryon. To describe the compact D4 brane, the background metric (1) is rewritten as

$$
d s^{2}=\left(\frac{U}{R}\right)^{3 / 2}\left[-d t^{2}+d \mathbf{x}^{2}+f(U) d x_{4}^{2}\right]+\left(R^{3} U\right)^{1 / 2}\left(\frac{d \xi^{2}}{\xi^{2}}+d \theta^{2}+\sin ^{2} \theta d \Omega_{3}^{2}\right) .
$$

The world-volume coordinates of this compact D4 brane are $\left(t, \theta, \theta_{\alpha}\right)$ where $\theta_{\alpha}$ 's are the angular coordinates on $S^{3}$. The Dirac-Born-Infeld (DBI) action with Chern-Simons term for this brane is

$$
S_{D 4}=-\mu_{4} \int d^{5} \sigma e^{-\Phi} \sqrt{-\operatorname{det}\left(P\left[G_{p q}\right]+T^{-1} F_{p q}\right)}+\mu_{4} \int A_{(1)} \wedge F_{4} .
$$

$P[$ ] denotes the pull-back to the world-volume of D4 brane. The Hamiltonian for baryonic D4 branes comes from the Legendre transformed Lagrangian

$$
\mathcal{H}_{D 4}=\tau_{4} \int d \theta \sqrt{\left(\xi^{2}+\xi^{\prime 2}\right) \omega^{4 / 3}\left[D(\theta)^{2}+\sin ^{6} \theta\right]},
$$

where $\tau_{4}=\mu_{4} \Omega_{3} R^{3} U_{K K} /\left(2^{2 / 3} g_{s}\right)$ and $\xi=\xi(\theta)$ depends on only polar angle $\theta . D(\theta)$ is the displacement defined from the Lagrangian and solving the equation of motion (EOM) gives its form explicitly as

$$
D(\theta)=-2+\cos \theta\left(3-\cos ^{2} \theta\right) .
$$

Now let's turn to the focus on D6 branes. There exist the fundamental strings which is connected between D4 baryon and D6 probe brane. The end points of these strings on D6 branes play the role of the source of $U(1)$ gauge field on the branes. The spherical part of the background metric (1) could be rewritten.

$$
d s^{2}=\left(\frac{U}{R}\right)^{3 / 2}\left[-d t^{2}+d \mathbf{x}^{2}+f(U) d x_{4}^{2}\right]+\left(\frac{R}{U}\right)^{3 / 2}\left(\frac{U}{\xi}\right)^{2}\left(d r^{2}+r^{2} d \Omega_{2}^{2}+d y^{2}+y^{2} d \phi^{2}\right)
$$


The D6 brane spans through the coordinates $\left(t, \mathbf{x}, r, \theta_{a}\right)$ where $\theta_{a}$ is the angular coordinate on $S^{2}$. The DBI action for D6 branes is

$$
S_{D 6}=-\mu_{6} \int d^{7} \sigma e^{-\Phi} \sqrt{-\operatorname{det}\left(P\left[G_{p q}\right]+T^{-1} F_{p q}\right)} .
$$

Also the Hamiltonian is given through the Legendre transformation as

$$
\mathcal{H}_{D 6}=\tau_{6} \int d r \sqrt{\left(1+\left(y^{\prime}\right)^{2}\right) \omega^{4 / 3}\left(\omega^{8 / 3} r^{4}+\tilde{Q}^{2}\right)}
$$

where $\tau_{6}=\mu_{6} \Omega_{2} V_{3} U_{K K}^{3} /\left(4 g_{s}\right)$ and the dimensionless quantity $\tilde{Q}$ is related to the number of fundamental string $Q$ by $\tilde{Q}=Q U_{K K} /\left(2^{5 / 3} \pi \alpha^{\prime} \tau_{6}\right)$. The tension of the fundamental string is always bigger than that of the brane. So the string itself shrinks and D4 and D6 branes are touched at a cusp eventually. In order that the configurations become stable, pulling down- and pushing up- force at the cusp should be the same. This is called the force balancing condition (FBC). The force at the cusp is obtained from the derivative of the Hamiltonian $\mathcal{H}$ with respect to the position of the cusp $\xi_{c}$ (or $U_{c}$ ).

$$
\left.F \equiv \frac{\partial \mathcal{H}}{\partial U_{c}}\right|_{\text {other values are fixed }}
$$

Then the FBC is expressed as

$$
\frac{Q}{N_{c}} F_{D 4}=F_{D 6}^{+}\left(Q_{+}\right)+F_{D 6}^{-}\left(Q_{-}\right)
$$

where $Q_{+}=\tilde{\alpha} Q$ and $Q_{-}=(1-\tilde{\alpha}) Q$. The super-(or sub-)script character $+/-$ denote the quantities of upper and lower branes respectively. $\tilde{\alpha}$ plays the role of the asymmetry parameter; $\tilde{\alpha}=0$ or 1 means the maximally asymmetric state and $\tilde{\alpha}=1 / 2$ corresponds to symmetric one. For a given total charge $Q$, there are a lot of embedding configurations which satisfy the FBC. The stable configuration is determined as one which gives the minimum energy. The total energy is given as the sum of the Hamiltonians of D4 baryonic vertices and two D6 branes.

$$
E_{\text {tot }}=\frac{Q}{N_{c}} \mathcal{H}_{D 4}+\mathcal{H}_{D 6}^{+}\left(Q_{+}\right)+\mathcal{H}_{D 6}^{-}\left(Q_{-}\right)
$$

Fig. 1 is one example of our results [17]. In low density region, the fundamental string is attached only to the lower brane $(\tilde{\alpha}=0)$. As the density increases, it starts to touch the upper brane. For very large density, as expected, $\tilde{\alpha}$ is close to $1 / 2$. Extremely dense matter does not distinguish the quarks with different masses, so becomes symmetric.

\section{Symmetry energy}

The nuclear symmetry energy is defined as the energy per nucleon which is needed to change the symmetric nuclear matter $\left(N_{p}=N_{n}\right)$ to the pure neutron matter $\left(N_{p}=0\right)$. The usual asymmetric factor $\alpha \equiv\left(N_{p}-N_{n}\right) /\left(N_{p}+N_{n}\right)$ is useful to describe the symmetry energy. In general the energy per nucleon can be approximately written as

$$
E(\rho, \alpha)=E(\rho, 0)+E_{\text {sym }}(\rho) \alpha^{2}+O\left(\alpha^{4}\right),
$$

where

$$
\left.E_{\text {sym }}(\rho) \equiv \frac{1}{2} \frac{\partial^{2} E}{\partial \alpha^{2}}\right|_{\alpha=0}
$$

is the symmetry energy and $\rho$ is the nucleon number density. 


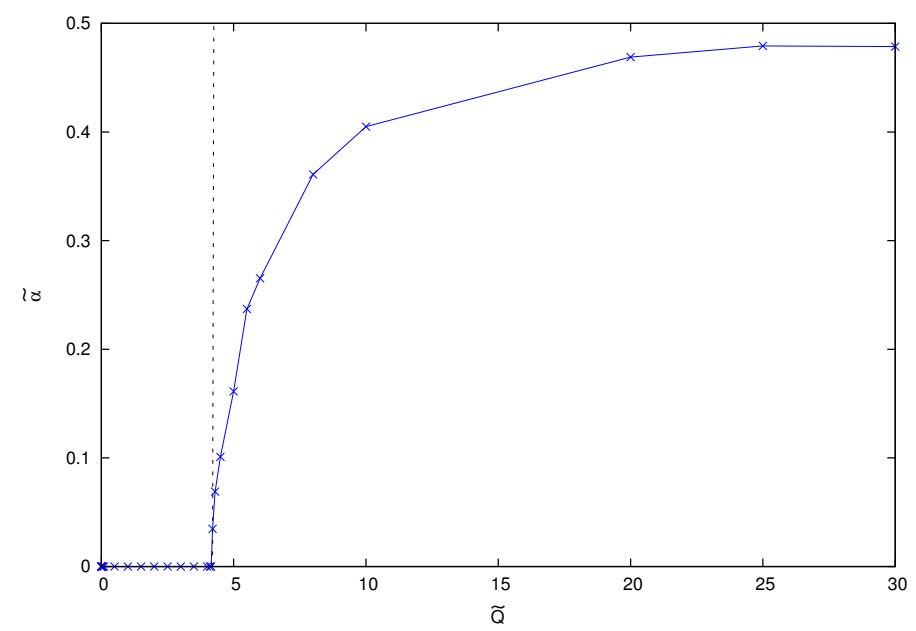

Fig. 1. Density dependence of $\tilde{\alpha}$. In low density $\left(\tilde{Q}<\tilde{Q}_{c}\right)$, the only lower brane is considered $(\tilde{\alpha}=0)$. As the density increases, the upper brane also touches D4 brane $(\tilde{\alpha} \neq 0)$. Here the transition occurs at $\tilde{Q}_{c}=4.2$.

The total energy $E_{t o t}$ in our model is given in eq. (13). If we take the configuration with same mass $\left(m_{+}=m_{-}\right)$, then symmetry energy can be expressed explicitly as

$$
E_{\text {sym }}(\tilde{Q})=\frac{2 \tau_{6}}{N_{B}} \int d r \frac{\sqrt{1+\left(y^{\prime}\right)^{2}} \tilde{Q}^{2} \omega^{10 / 3} r^{4}}{\left(\tilde{Q}^{2}+4 \omega^{8 / 3} r^{4}\right)^{3 / 2}}
$$

Here $N_{B}=Q / N_{c}$ is the number of baryons and we use the embedding $y(r)$ obtained from the EOM with a condition $m_{+}=m_{-}$. To calculate the symmetry energy in our model, we use the relation $\tilde{\alpha}=(1-\alpha) / 2$. The experimental results for the symmetry energy are summed up as

$$
E_{\text {sym }}(\rho)=c\left(\frac{\rho}{\rho_{0}}\right)^{\gamma}
$$

where $\rho_{0}$ is the normal nuclear density. In low density regime, $0.3 \lesssim \rho / \rho_{0} \lesssim 1$, for example, $c \simeq$ $31.6 \mathrm{MeV}$ and $\gamma=0.5 \sim 0.7$ [5]. In our calculations, with the choice of $\lambda=6$ and $M_{K K}=1.04 \mathrm{GeV}$, $\gamma \simeq 0.5$ and $c \simeq 27 \mathrm{MeV}$. The value of $c$ depends on the parameter, while $\gamma$ is rather universal. Here we have used the quarks with same masses, for simplicity. Our result is shown in Fig. 2. For more detailed description, see [19].

\section{Meson mass in asymmetric matter}

In holographic QCD, the mesons correspond to the excitation mode of strings whose both end points are attached at the branes. These modes can be described as the fluctuations of branes. To see the behavior of the meson mass in asymmetric dense matter, the fluctuations of the probe branes obtained in [17] are considered.

We start from the non-Abelian DBI action [20].

$$
S_{D 6}^{n A}=-\mu_{6} \int d^{7} \sigma \operatorname{STr}\left[e^{-\Phi} \sqrt{-\operatorname{det}\left(P\left[G_{p q}+G_{p a}\left(Q^{-1}-\delta\right)^{a b} G_{b q}\right]+T^{-1} F_{p q}\right)} \sqrt{\operatorname{det} Q_{b}^{a}}\right]
$$

STr means the symmetrized trace for flavor indices and is introduced to avoid the ordering-ambiguity [21]. The matrix $Q_{b}^{a}$ is defined as

$$
Q_{b}^{a} \equiv \delta_{b}^{a}+i T\left[X^{a}, X^{c}\right] G_{c b}
$$




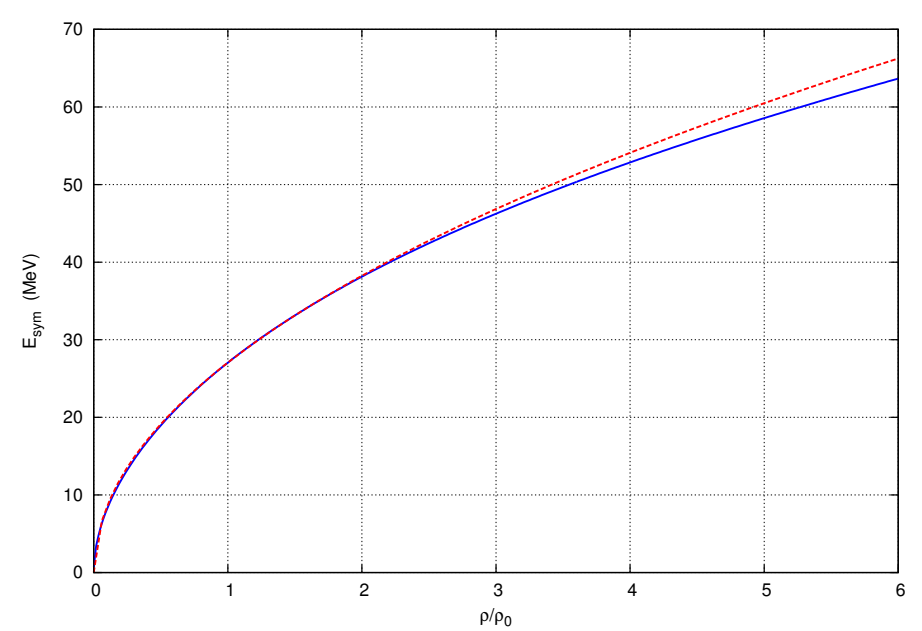

Fig. 2. Density dependence of the symmetry energy $E_{\text {sym }}$. Solid line is the symmetry energy from the model. The dotted one is for $E_{\text {sym }}=c \sqrt{\rho / \rho_{0}}$. For illustration purpose, we take $\lambda=6$ and $M_{K K}=1.04 \mathrm{GeV}$. The result is fitted to the curve with $c \sim 27 \mathrm{MeV}$.

where $X^{a}$ 's are the coordinates transverse to D6 branes. We will take an ansatz of diagonal embeddings [22]. Then, with the diagonal background metric $G_{m n}$, the above action is expanded up to $O\left(X^{4}\right)$ as

$$
\begin{array}{r}
S_{D 6}^{n A}=-\mu_{6} \int d^{7} \sigma \operatorname{STr}\left[e^{-\Phi} \sqrt{-\operatorname{det}\left\{G_{p q}+T^{-1} F_{p q}+D_{p} X^{a} D_{q} X^{b}\left(G_{a b}-i T\left[X^{c}, X^{d}\right] G_{a c} G_{d b}\right)\right\}}\right. \\
\left.\times\left(1+\frac{T^{2}}{4}\left[X^{a}, X^{b}\right] G_{b c}\left[X^{c}, X^{d}\right] G_{d a}\right)\right] .
\end{array}
$$

For simplicity we will consider the case of $N_{f}=2$. Then all fields are expressed by $2 \times 2$-matrices. In this case Pauli matrices is useful.

$$
M=M_{0} \tau^{0}+M_{i} \tau^{i}=\frac{1}{2}\left(\begin{array}{ll}
M_{0}+M_{3} & M_{1}-i M_{2} \\
M_{1}+i M_{2} & M_{0}-M_{3}
\end{array}\right)=\left(\begin{array}{cc}
M_{+} & M_{12} \\
M_{21} & M_{-}
\end{array}\right)
$$

We will use the classical solutions that have been obtained in Sec. 2. As stated, the fluctuations are considered as the holographic duals of mesons.

$$
X^{y}=y+x^{y}, \quad X^{\phi}=0+\varphi \quad \text { and } \quad A=\bar{A}+\alpha
$$

We can easily check that the field $\varphi$, which is interpreted as Goldstone boson, decouples from the others. Let's focus only on this fluctuation. Then the DBI action is expanded up to quadratic order for $\varphi$ as

$$
S_{\varphi}=-\mu_{6} \int d^{7} \sigma \operatorname{STr}\left[e^{-\Phi} \sqrt{-\operatorname{det}\left(a^{(0)}\right)}\left(1+\frac{1}{2} \operatorname{tr}\left[\left(a^{(0)}\right)^{-1} a^{(2)}\right]+\frac{T^{2}}{4}\left[X^{a}, X^{b}\right] G_{b c}\left[X^{c}, X^{d}\right] G_{d a}\right)\right],
$$

where $a^{(0)}$ is the induced background metric itself with 0th order fluctuation and

$$
\begin{aligned}
a_{p q}^{(2)}=G_{\phi \phi}\left(\partial_{p} \varphi\right. & \left.+i\left[\bar{A}_{p}, \varphi\right]\right)\left(\partial_{q} \varphi+i\left[\bar{A}_{q}, \varphi\right]\right) \\
& -i T G_{y y} G_{\phi \phi}\left\{\partial_{p} y\left(\partial_{q} \varphi+i\left[\bar{A}_{q}, \varphi\right]\right)-\left(\partial_{p} \varphi+i\left[\bar{A}_{p}, \varphi\right]\right) \partial_{q} y\right\}[y, \varphi] .
\end{aligned}
$$

Then, after taking a symmetrized trace, we can read off the Lagrangian for this field $\varphi$ up to quadratic order [23].

$$
\mathcal{L}_{\varphi_{ \pm}}^{(2)}=\frac{1}{2}\left[\bar{F} D^{-1}\left\{G_{r r}+G_{y y}\left(y^{\prime}\right)^{2}\right\} G_{\phi \phi}\right]_{y_{ \pm}}\left(\dot{\varphi_{ \pm}}\right)^{2}+\frac{1}{2}\left[\bar{F} D^{-1} G_{t t} G_{\phi \phi}\right]_{y_{ \pm}}\left(\varphi_{ \pm}{ }^{\prime}\right)^{2}
$$


EPJ Web of Conferences

$$
\begin{aligned}
& \mathcal{L}_{\varphi_{i n t}}^{(2)}=\frac{1}{2}\left(\left[\bar{F} D^{-1}\left\{G_{r r}+G_{y y}\left(y^{\prime}\right)^{2}\right\} G_{\phi \phi}\right]_{y_{+}}+\left[\bar{F} D^{-1}\left\{G_{r r}+G_{y y}\left(y^{\prime}\right)^{2}\right\} G_{\phi \phi}\right]_{y_{-}}\right) \\
& \times\left\{\dot{\varphi}_{12} \dot{\varphi}_{21}+i A_{3}\left(\varphi_{12} \dot{\varphi_{21}}-\dot{\varphi}_{12} \varphi_{21}\right)+\left(A_{3}\right)^{2} \varphi_{12} \varphi_{21}\right\} \\
& -\frac{i y_{3}}{2}\left(\left[\bar{F} D^{-1} G_{y y} G_{\phi \phi} A^{\prime} y^{\prime}\right]_{y_{+}}+\left[\bar{F} D^{-1} G_{y y} G_{\phi \phi} A^{\prime} y^{\prime}\right]_{y_{-}}\right)\left(\varphi_{12} \dot{\varphi}_{21}-\dot{\varphi_{12}} \varphi_{21}-2 i A_{3} \varphi_{12} \varphi_{21}\right) \\
& +\frac{1}{2}\left(\left[\bar{F} D^{-1} G_{t t} G_{\phi \phi}\right]_{y_{+}}+\left[\bar{F} D^{-1} G_{t t} G_{\phi \phi}\right]_{y_{-}}\right) \varphi_{12}{ }^{\prime} \varphi_{21}^{\prime} \\
& +\frac{\left(y_{3}\right)^{2}}{2}\left(\left[T^{2} \bar{F} G_{y y} G_{\phi \phi}\right]_{y_{+}}+\left[T^{2} \bar{F} G_{y y} G_{\phi \phi}\right]_{y_{-}}\right) \varphi_{12} \varphi_{21} \\
& =\frac{1}{2} \mathbb{P}\left\{\dot{\varphi_{12}} \dot{\varphi_{21}}+i A_{3}\left(\varphi_{12} \dot{\varphi_{21}}-\dot{\varphi}_{12} \varphi_{21}\right)+\left(A_{3}\right)^{2} \varphi_{12} \varphi_{21}\right\} \\
& -\frac{i y_{3}}{2} \mathbb{Q}\left(\varphi_{12} \dot{\varphi}_{21}-\dot{\varphi_{12}} \varphi_{21}-2 i A_{3} \varphi_{12} \varphi_{21}\right)+\frac{1}{2} \mathbb{R} \varphi_{12}{ }^{\prime} \varphi_{21}{ }^{\prime}+\frac{\left(y_{3}\right)^{2}}{2} \mathbb{S} \varphi_{12} \varphi_{21}
\end{aligned}
$$

Here we introduced the common expression $\bar{F}$ as

$$
\bar{F} \equiv e^{-\Phi} \sqrt{-\operatorname{det}\left(a^{(0)}\right)}
$$

and the dotted and primed variables correspond to the time- and radial- derivatived one respectively.

The EOMs for $\varphi_{+}$and $\varphi_{-}$are trivial. The quadratic Lagrangian of each field contains only single embedding solution $y_{+}$or $y_{-}$, and so its EOM is very similar to that of the single brane case.

$$
0=\mathbb{P}_{ \pm} \ddot{\varphi_{ \pm}}+\mathbb{R}_{ \pm} \varphi_{ \pm}^{\prime \prime}+\mathbb{R}_{ \pm}^{\prime} \varphi_{ \pm}^{\prime}
$$

If we decompose $\varphi$ as

$$
\varphi(t, r)=e^{-i \omega t} \Phi(r)
$$

then above equation can be written as

$$
0=\mathbb{R}_{ \pm} \Phi_{ \pm}^{\prime \prime}+\mathbb{R}_{ \pm}^{\prime} \Phi_{ \pm}^{\prime}-\omega_{ \pm}^{2} \mathbb{P}_{ \pm} \Phi_{ \pm} .
$$

The off-diagonal components, on the other hand, are more complicated. Those depend on both brane configurations naturally.

$$
\begin{aligned}
& 0=\mathbb{P} \ddot{\varphi_{12}}+2 i\left(A_{3} \mathbb{P}-y_{3} \mathbb{Q}\right) \dot{\varphi_{12}}+\mathbb{R} \varphi_{12}^{\prime \prime}+\mathbb{R}^{\prime} \varphi_{12}^{\prime}+\left\{-\left(A_{3}\right)^{2} \mathbb{P}+2 y_{3} A_{3} \mathbb{Q}-\left(y_{3}\right)^{2} \mathbb{S}\right\} \varphi_{12} \\
& 0=\mathbb{P} \ddot{\varphi_{21}}-2 i\left(A_{3} \mathbb{P}-y_{3} \mathbb{Q}\right) \dot{\varphi}_{21}+\mathbb{R} \varphi_{21}^{\prime \prime}+\mathbb{R}^{\prime} \varphi_{21}^{\prime}+\left\{-\left(A_{3}\right)^{2} \mathbb{P}+2 y_{3} A_{3} \mathbb{Q}-\left(y_{3}\right)^{2} \mathbb{S}\right\} \varphi_{21}
\end{aligned}
$$

These equations are also decomposed as

$$
\begin{aligned}
& 0=\mathbb{R} \Phi_{12}^{\prime \prime}+\mathbb{R}^{\prime} \Phi_{12}^{\prime}-\left\{\left(\omega_{12}-A_{3}\right)^{2} \mathbb{P}+2 y_{3}\left(\omega_{12}-A_{3}\right) \mathbb{Q}+\left(y_{3}\right)^{2} \mathbb{S}\right\} \Phi_{12} \\
& 0=\mathbb{R} \Phi_{21}^{\prime \prime}+\mathbb{R}^{\prime} \Phi_{21}^{\prime}-\left\{\left(\omega_{21}+A_{3}\right)^{2} \mathbb{P}-2 y_{3}\left(\omega_{21}+A_{3}\right) \mathbb{Q}+\left(y_{3}\right)^{2} \mathbb{S}\right\} \Phi_{21} .
\end{aligned}
$$

We solve these EOMs numerically and the results for $\varphi_{12}$ and $\varphi_{21}$ are given in Fig. 3 [23]. In low density, the masses of $\varphi_{12}$ and $\varphi_{21}$ split due to the effect of the isospin chemical potential in $A_{3}$. As the density increases, the density effect becomes dominant and so both masses start to decrease. Beyond the critical value $\tilde{Q}_{c}$, the effect of asymmetry in $A_{3}$ is smaller than that of the density, and the difference between two modes is negligible.

Since these off-diagonal component modes correspond to the meson made up of two quarks with different masses, they can be considered as $K^{ \pm}$. The mass behavior of our model is in agreement with the results in [24].

\section{Summary}

We calculated some quantities using the holographic QCD model to study the some properties of asymmetric dense matter. One of the typical quantities of asymmetric matter is the nuclear symmetry 


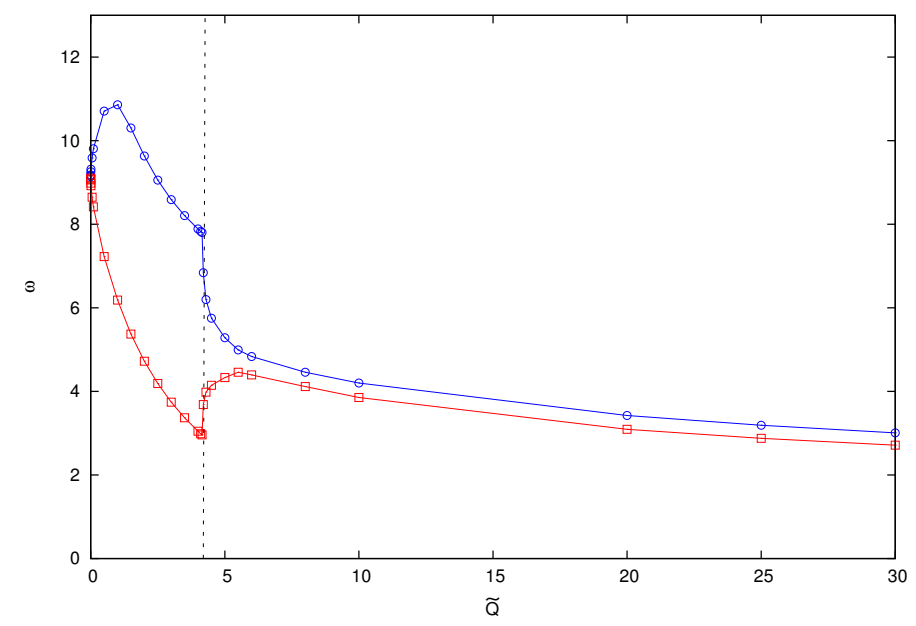

Fig. 3. The mass spectrum of the (pseudo-)scalar mesons, $\varphi_{12}$ and $\varphi_{21}$

energy. The behavior of symmetry energy obtained in D4/D6/D6 model is similar to the experimental results at least below the nuclear matter density $\rho_{0}$ [19]. Another interesting behavior we can see in dense matter is hadron mass splitting. Mesons composed of the quarks with different masses can be represented by the fluctuation of $\mathrm{D}$ branes with different asymptotic heights. We can also find similar mass splitting in the asymmetric matter in this model [23]. The holographic QCD might be helpful to catch main feature of the physics of asymmetric matter.

\section{Acknowledgment}

YK and IJS acknowledge the Max Planck Society (MPG), the Korea Ministry of Education, Science and Technology (MEST), Gyeongsangbuk-Do and Pohang City for the support of the Independent Junior Research Group at the Asia Pacific Center for Theoretical Physics (APCTP).

\section{References}

1. P. Danielewicz, R. Lacey and W. G. Lynch, Science 298, 1592 (2002) [arXiv:nucl-th/0208016].

2. A. W. Steiner, M. Prakash, J. M. Lattimer and P. J. Ellis, Phys. Rept. 411, 325 (2005) [arXiv:nuclth/0410066].

3. B. A. Li, L. W. Chen and C. M. Ko, Phys. Rept. 464, 113 (2008) [arXiv:0804.3580 [nucl-th]].

4. C. Xu and B. A. Li, Phys. Rev. C 81, 064612 (2010) [arXiv:0910.4803 [nucl-th]].

5. D. V. Shetty and S. J. Yennello, Pramana 75, 259 (2010) [arXiv:1002.0313 [nucl-ex]].

6. M. Di Toro, V. Baran, M. Colonna and V. Greco, J. Phys. G 37, 083101 (2010) [arXiv:1003.2957 [nucl-th]].

7. H. K. Lee, B. Y. Park and M. Rho, Phys. Rev. C 83, 025206 (2011) [arXiv:1005.0255 [nucl-th]].

8. D. T. Son and M. A. Stephanov, Phys. Rev. Lett. 86, 592 (2001) [arXiv:hep-ph/0005225].

9. J. Rizzo, M. Colonna, M. Di Toro and V. Greco, Nucl. Phys. A 732, 202 (2004) [arXiv:nuclth/0309032].

10. U. G. Meissner, A. M. Rakhimov, A. Wirzba and U. T. Yakhshiev, Eur. Phys. J. A 32, 299 (2007) [arXiv:0705.1603 [nucl-th]].

11. J. M. Maldacena, Adv. Theor. Math. Phys. 2, 231 (1998) [Int. J. Theor. Phys. 38, 1113 (1999)] [arXiv:hep-th/9711200]. 
12. S. S. Gubser, I. R. Klebanov and A. M. Polyakov, Phys. Lett. B 428, 105 (1998) [arXiv:hepth/9802109].

13. E. Witten, Adv. Theor. Math. Phys. 2, 253 (1998) [arXiv:hep-th/9802150].

14. M. Kruczenski, D. Mateos, R. C. Myers and D. J. Winters, JHEP 0405, 041 (2004) [arXiv:hepth/0311270].

15. T. Sakai and S. Sugimoto, Prog. Theor. Phys. 113, 843 (2005) [arXiv:hep-th/0412141].

16. J. Erlich, E. Katz, D. T. Son and M. A. Stephanov, Phys. Rev. Lett. 95, 261602 (2005) [arXiv:hep$\mathrm{ph} / 0501128]$.

17. Y. Kim, Y. Seo and S. J. Sin, JHEP 1003, 074 (2010) [arXiv:0911.3685 [hep-th]].

18. E. Witten, JHEP 9807, 006 (1998) [arXiv:hep-th/9805112].

19. Y. Kim, Y. Seo, I. J. Shin and S. J. Sin, JHEP 1106, 011 (2011) [arXiv:1011.0868 [hep-ph]].

20. R. C. Myers, JHEP 9912, 022 (1999) [arXiv:hep-th/9910053].

21. A. A. Tseytlin, Nucl. Phys. B 501 (1997) 41 [arXiv:hep-th/9701125].

22. J. Erdmenger, K. Ghoroku and I. Kirsch, JHEP 0709, 111 (2007) [arXiv:0706.3978 [hep-th]].

23. Y. Kim, Y. Seo, I. J. Shin and S. J. Sin, "Holographic meson mass in asymmetric dense matter," in preparation.

24. J. Schaffner and I. N. Mishustin, Phys. Rev. C 53, 1416 (1996) [arXiv:nucl-th/9506011]. 